\title{
Ultrafast diffusion at the onset of growth: $\mathrm{O} / \mathrm{Ru}(0001)$
}

\author{
Jack Kelsall, ${ }^{1,}$ | $P e t e r$ S. M. Townsend,${ }^{2}$ John Ellis,${ }^{1}$ Andrew P. Jardine,${ }^{1}$ and Nadav Avidor ${ }^{1},{ }^{\dagger}$ \\ ${ }^{1}$ Cavendish Laboratory, University of Cambridge, \\ $19 \mathrm{~J} J$ Thomson Avenue, Cambridge CB3 OHE, United Kingdom \\ ${ }^{2}$ Department of Chemistry and Chemical Biology, \\ Rutgers University, Piscataway, New Jersey 08854, USA
}

(Dated: March 8, 2021)

\begin{abstract}
Nanoscopic clustering in a 2D disordered phase is observed for oxygen on $\mathrm{Ru}(0001)$ at low coverages and high temperatures. We study the coexistence of quasi-static clusters (with a characteristic length of $\sim 9 \AA$ ) and highly mobile atomic oxygen which diffuses between the energy-inequivalent, threefold hollow sites of the substrate. We determine a surprisingly low activation energy for diffusion of $385 \pm 20 \mathrm{meV}$. The minimum of the $\mathrm{O}-\mathrm{O}$ interadsorbate potential appears to be at lower separations than previously reported.
\end{abstract}

The nanoscopic mechanisms underpinning twodimensional (2D) growth at surfaces are increasingly of interest as layered materials are explored and exploited for applications [1 5]. Of particular importance is the local, coverage-dependent, dynamic equilibrium between the diffusing precursors and the clusters that develop from nucleation events at the onset of growth. Attractive interadsorbate interactions, which induce islanding, can be studied most readily at low surface coverage and elevated temperatures. These high temperatures also facilitate the growth of high-quality 2D materials [6, 7].

The surface diffusion of atomic oxygen is a key process in applications such as water splitting for hydrogenbased clean energy $[8]$ and the realization of free-standing graphene 9. On certain transition metals from the platinum group, oxygen exhibits multiple stable surface phases but does not form oxides under typical ultrahigh vacuum (UHV) conditions [10, 11. The nature of the structures formed suggests that the interaction between $\mathrm{O}$ adatoms cannot be successfully modeled as simple monotonic repulsion, which has been relatively well characterized by diffusion measurements [12 14. $\mathrm{O} /$ transition metal systems are therefore excellent candidates for studying more complicated models of interadsorbate forces.

Previous work on the ordered phases of $\mathrm{O} / \mathrm{Ru}(0001)$ at coverages of $\theta=0.25$ and 0.5 ML has proceeded mainly via low-energy electron diffraction (LEED) investigations at room temperature [10, 15, 16. Under these conditions, the adatoms are observed to exclusively occupy hcp hollow sites on the hexagonal close-packed $\mathrm{Ru}(0001)$ surface. The authors conclude that $\mathrm{O} / \mathrm{Ru}(0001)$ can be described as a lattice gas dominated by short-range interactions. However, at these relatively high coverages, it is only possible to estimate the interaction potential at separations up to twice the substrate lattice constant, $2 a$.

Scanning tunneling microscope (STM) studies have reported on clustering and interadsorbate interactions in

\footnotetext{
*jck40@cam.ac.uk

$\dagger$ na364@cam.ac.uk
}

low-coverage $\mathrm{O} / \mathrm{Ru}(0001)$ [17, 18. The islanding of oxygen atoms separated by $2 a$ is visible in these STM images. The investigations took place at room temperature so the jump rate was sufficiently low for STM measurements to resolve motion. As a result, occupation of the fcc hollow sites is negligible, meaning that certain aspects of the nanoscopic dynamics cannot be studied. The mean residence time of isolated oxygen atoms is found to be $60 \mathrm{~ms}$, corresponding to a diffusion barrier between $0.55 \mathrm{eV}$ and $0.7 \mathrm{eV}$. Renisch et al. have also measured the $\mathrm{O}-\mathrm{O}$ dimer lifetime to be $220 \mathrm{~ms}$ and deduced a potential well depth of $\approx 50 \mathrm{meV}$ for a separation of $2 a$ [18. Overall, the results suggest a fine balance between thermal fluctuations and attractive interactions, making $\mathrm{O} / \mathrm{Ru}(0001)$ an ideal candidate in which to study adsorbate dynamics in the vicinity of clusters.

In this work, we study low-coverage atomic diffusion of oxygen on $\mathrm{Ru}(0001)$ at elevated temperatures using ${ }^{3} \mathrm{He}$ spin echo spectroscopy $\left({ }^{3} \mathrm{HeSE}\right)$, demonstrating our ability to measure surface dynamics in a regime inaccessible to other techniques [19. We determine residence times for jump diffusion of order tens of picoseconds, around $10^{8}$ times faster than previous experiments. Measuring at high temperatures allows us to resolve the occupation of the higher-energy fcc hollow site for the first time, in addition to enabling us to determine the Arrhenius pre-exponential factor at near-desorption temperatures. Moreover, we observe quasi-static oxygen islands despite the fact that no long-range ordered phases are thermodynamically stable at these temperatures, at any coverage. We obtain a time-resolved diffraction scan by interpreting the ${ }^{3} \mathrm{HeSE}$ data in terms of jump diffusion models, thus avoiding the inelastic background broadening which is inherent in conventional diffraction.

All data were obtained using the Cambridge ${ }^{3} \mathrm{HeSE}$ spectrometer, in UHV [20, 21]. When utilized to study dynamics of surfaces, the instrument measures the total intermediate scattering function (ISF), $I(\Delta \mathbf{K}, t)$, of the surface. $\Delta \mathbf{K}$ is the surface-parallel component of the momentum transfer between an incoming ${ }^{3} \mathrm{He}$ atom and the surface. $t$ is the correlation time of the measurement. $I(\Delta \mathbf{K}, t)$ is the spatial Fourier transform of the coherent 
van Hove correlation function, $G(\mathbf{R}, t)$, which contains all the details of the microscopic motion occurring [21]. For a given incident beam energy distribution, centered on $8.0 \mathrm{meV}$ for the measurements here, $\Delta \mathbf{K}$ is varied by altering the orientation of the sample. The length scale probed in the scattering direction is $2 \pi /|\Delta \mathbf{K}|$. Thus, the instrument permits nanoscopic measurements of diffusion and correlated motion. The crystal used for the duration of the experiment was cleaned until a ${ }^{3} \mathrm{He}$ reflectivity of at least $20 \%$ (at $400 \mathrm{~K}$ ) was obtained, indicating a sufficiently clean and flat surface. Helium diffraction scans were obtained after each cleaning cycle to confirm surface quality (see Supplementary Information, SI, section 1 for further details).

We obtained diffusion measurements in the range 750$900 \mathrm{~K}$ to investigate both the mechanism of motion and the temperature dependence of the diffusion rate. The high temperatures mean we expect no contamination of the sample from UHV residual gases, e.g. carbon monoxide and hydrogen [22, 23. A coverage of $\theta=0.09 \mathrm{ML}$ was used throughout the measurement process. The calculation of coverage is outlined in SI section 2 .

Typically, ISFs oscillate over short times due to periodic motion, e.g. intracell motion or phonons. Beyond a few picoseconds, when aperiodic motion dominates, the ISF decays monotonically. For jump diffusion on a lattice, a measured ISF has the functional form $F=A f+C$. Here, $f$ is a decaying function of time describing the motion, according to a particular model. Both $A$, the decay amplitude, and $C$, the background term related to the elastically scattered signal, contain information about the system and are discussed below. For the case of a Bravais lattice, $f_{1}=e^{-\alpha_{1} t} . \alpha_{1}$ is termed the dephasing rate. In the absence of interactions, the Chudley-Elliott (CE) model provides a simple analytic form for the rate, $\alpha_{1}(\Delta \mathbf{K})=2 \sum_{\mathbf{j}} \Gamma_{\mathbf{j}} \sin ^{2}(\Delta \mathbf{K} \cdot \mathbf{j} / 2)$ 24], where $\Gamma_{\mathbf{j}}$ is the mean rate of jumps that translate an adsorbate by the vector $\mathbf{j}$. Despite its simplicity, the model typically explains the overall shape of a measured $\alpha(\Delta \mathbf{K})$, even when only single jumps are included [21].

When diffusion occurs atop a lattice with two adsorption sites per primitive unit cell, the ISF is generally well-modeled by a biexponential function of the form $F_{2}=A_{1} e^{-\alpha_{1} t}+A_{2} e^{-\alpha_{2} t}+C$ with $\alpha_{2}>\alpha_{1}$. For the case of jump diffusion on a hexagonal lattice, the non-interacting hcp/fcc jump model of Tuddenham et al. provides explicit analytic expressions for the ratio of the amplitudes $A_{2} / A_{1}$ and for the two dephasing rates, in terms of just two parameters [25]. These quantities are $\tau$, the minority fcc site residence time, and $\lambda$, a measure of the energetic inequivalence between sites and defined such that the majority hcp residence time parameter is $\lambda \tau$. We refer to the unscaled model predicted by the theory as $f_{H}=f_{H}(\lambda, \tau ; \Delta \mathbf{K}, t)$. Interactions which induce correlations in the motion may cause the ISFs to deviate from analytic expectations. However, if the interactions are relatively weak, the ISFs are still well-described by exponentials and instead the dephasing rates are modified by

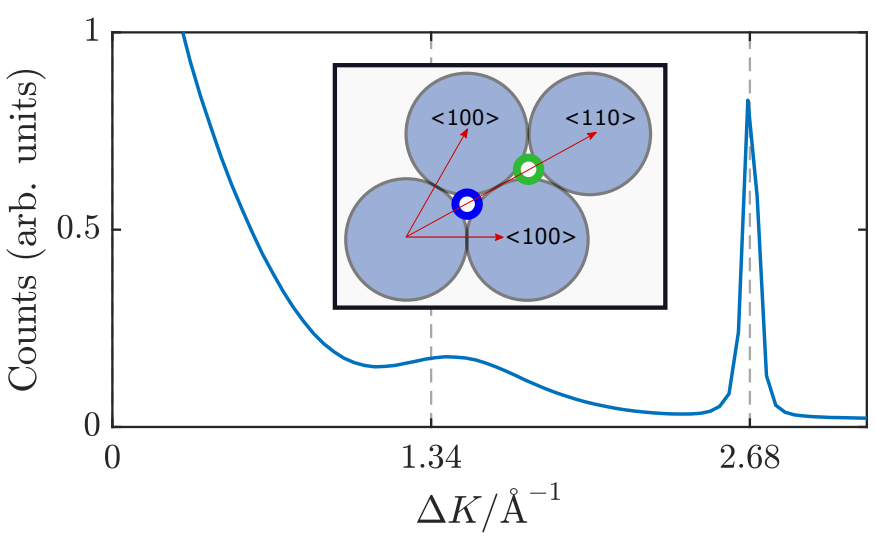

FIG. 1: Conventional diffraction scan of 0.09 ML $\mathrm{O} / \mathrm{Ru}(0001)$ at $850 \mathrm{~K}$. The narrow first-order peak is centered at $2.68 \AA^{-1}$. The peak at $\Delta K \approx 1.34 \AA^{-1}$,

which is entirely due to the adsorbed oxygen, is significantly broader and indicates that any adsorbate order is short-ranged. Inset: primitive unit cell of the $\mathrm{Ru}(0001)$ substrate; fcc and hcp adsorption sites are indicated by different-colored circles.

de Gennes features at values of $\Delta \mathbf{K}$ which correspond to preferred separation lengths [26, 27] and/or in a 'meanfield' fashion [28].

Figure 1 shows a typical diffraction scan obtained after dosing oxygen onto the $\mathrm{Ru}(0001)$ surface to a coverage of $\theta=0.09 \mathrm{ML}$, at $T=850 \mathrm{~K}$. Given that $T$ was greater than the order-disorder transition temperature at this $\theta$ [15], we expected no order in the adsorbed layer. However, a broad peak at the half-order position $\left(\Delta K \approx 1.34 \AA^{-1}\right)$ was always observed, in addition to the first-order peak. The diffraction can be attributed to clustering on $\mathrm{Ru}(0001)$ with local $p(2 \times 2)$ order.

Surface motion was investigated by recording a series of ISFs at $850 \mathrm{~K}$ from $\Delta K=0.05$ to $3.6 \AA^{-1}$ along the $\langle 110\rangle$ azimuth (see Figure 1). An example ISF is presented in Figure 2(c). To obtain an initial estimate of the dephasing rates, all points in each ISF were fitted to the function $F_{2}$. For a small number of ISFs, the amplitude of the fast exponential is low, as predicted by analytic models [25, meaning that the value of $\alpha_{2}$ cannot be estimated reliably. In such cases, the function $F_{1}$ was instead fitted to the data to obtain $\alpha_{1}$ only. In all cases, the first point of each ISF, at $t=0$, is excluded as it is influenced by short-time motion which is not relevant after a few picoseconds 21. The extracted dephasing rates are shown in Figure 2(a). We note that the fiveparameter fit to $F_{2}$ is somewhat sensitive to the initial estimate of the fit parameters, meaning that $\alpha_{1}$ and $\alpha_{2}$ must be treated as semi-quantitative estimates.

To determine the temperature dependence of the diffusion, we measured ISFs at $\Delta K=0.4 \AA^{-1}$ and $\Delta K=$ $0.9 \AA^{-1}$ between $750 \mathrm{~K}$ and $900 \mathrm{~K}$. Given the predominant jump models, which should be thermally activated, we expect Arrhenius behaviour, i.e. $\alpha \propto e^{-E / k_{B} T}$. $E$ 

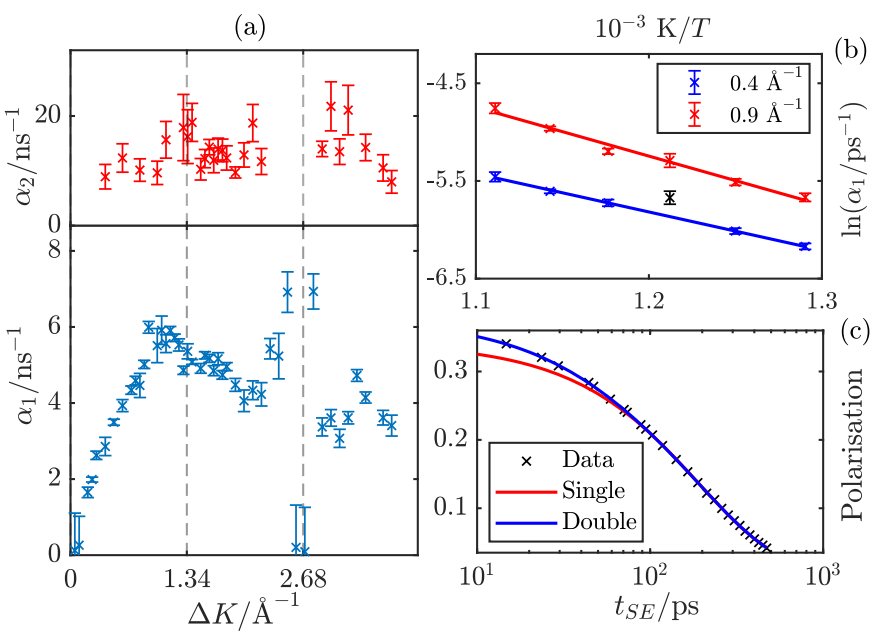

FIG. 2: (a) $\alpha(\Delta \mathbf{K})$ from fitting the $\langle 110\rangle$ experimental ISFs to $F_{1}$ and $F_{2}$. Near the edge of the first Brillouin zone (right vertical dashed line), the slow decay rate, $\alpha_{1}$, is expected to tend towards zero. The anomalous peak in $\alpha_{1}$ at that position can be attributed to a misfit

because the fast and slow components cannot be distinguished. (b) Arrhenius plots of $\alpha_{1}$ for two values of $\Delta K$ over a range of temperatures. The black point is excluded from the calculation of the second gradient as it was recorded at the end of a measurement cycle and may be affected by contaminants. (c) A typical ISF, corresponding to $\Delta K=1.5 \AA^{-1}$, alongside fits to $F_{1}$ and $F_{2}$.

is the effective energy barrier for diffusion [21. The long time 'tail' of these ISFs were fitted to $F_{1}$, without constraints, to extract the slow dephasing rate. Figure 2 (b) shows $\ln \left(\alpha_{1} / \mathrm{ps}\right)$ plotted against inverse temperature, from which we extract $E=385 \pm 20 \mathrm{meV}$. The value is expected to be slightly lower than the adiabatic potential energy barrier [21. Our value of $E$ is lower than previous estimates which were deduced via assumed (rather than measured) rate prefactors [17, 18. A rate prefactor of $\Gamma_{0}=0.56 \pm 0.2 \mathrm{ps}^{-1}$ has also been determined using a single-jump CE model to account for the $\Delta \mathbf{K}$-dependent part of each prefactor. $\Gamma_{0} e^{-E / k_{B} T}$ is thus the mean jump rate of an $\mathrm{O}$ atom to any of its six neighboring hcp sites.

We now analyse the dephasing rates. Comparison with previous measurements suggests that $\alpha_{2}$ (see Figure 2) cannot be attributed to the diffusion of a contaminant such as carbon [29]. The slow and fast dephasing rates in Figure 2 are qualitatively consistent with the hcp/fcc jump model $F_{H}$. It is expected that oxygen will undergo intracell diffusion by jumping between the majority hcp and minority fcc hollow sites, giving the additional fast exponential component present in $F_{H}$. Intracell motion can be resolved in the present case because the high temperatures means the occupation of metastable fcc sites is non-negligible. To test this hypothesis, and because noise means the independent biexponential fitting of each polarization scan does not always resolve a fast component, we have applied a marginalized global Bayesian analysis which utilizes the model $F_{H}$ [30]. In doing so, we progress to a constrained model $F_{H}$ which depends on fewer parameters and is thus a more discriminating test of the data. Applying a non-interacting model to interacting data in this way has previously been shown to give accurate estimates of surface parameters, providing that the interadsorbate forces are sufficiently weak, although the fit may be poor at certain values of $\Delta K$ [31. Given the low coverages and the absence of a strong de Gennes features in Figure 2, we make the same assumption here.

The Bayesian method evaluates the probability that the scaled model $F_{H}=A f_{H}+C$ explains a particular ISF. The amplitude $A$ and offset $C$ are integrated over (marginalized) by the method, while different values of $\lambda$ and $\tau$ are tested. The method is 'global' as the calculated probabilities are subsequently multiplied together to obtain a probability matrix in $(\lambda, \tau)$ space representing the whole dataset. The resulting plot is given in Figure 3(a). The most likely values of $\lambda=9.5 \pm 1$ and $\tau=14.3 \pm 2 \mathrm{ps}$ are derived from its maximum. The uncertainties have been estimated using the dimensions of the 0.68 relative probability contour; however, the errors are not independent as the principal axes at the maximum of the surface in Figure 3 (a) are not parallel to the $\lambda$ and $\tau$ axes. The parameter $\lambda$ can be converted to an energy inequivalence $\Delta E$ via the approximate relationship $\lambda=e^{\Delta E / k_{B} T} 25$. The CE model would be a good description of the dataset in the $\lambda \rightarrow \infty$ limit. The value of $\Delta E=165 \pm 9 \mathrm{meV}$ obtained is significantly smaller than previous density functional theory (DFT) estimates [32, 33. The discrepancy may result in part from the zero-point-energy of a particle occupying a hcp site relative to the fcc site. Furthermore, the DFT results, obtained at $0 \mathrm{~K}$, may not accurately describe the $\mathrm{Ru}(0001)$ surface at $\sim 850 \mathrm{~K}$. The sharply-peaked distribution of Figure 3(a) is an indication that the model successfully describes most of the experimental ISFs, despite the interactions between adatoms. We therefore conclude that the diffusion of isolated (i.e. non-clustered) atoms dominates the diffusion signature.

More information can be extracted by taking the parameters $\lambda=9.5 \pm 1$ and $\tau=14.3 \pm 2 \mathrm{ps}$ as inputs to the model $F_{H}$ and comparing it to individual experimental ISFs. We fit the dataset to $F_{H}$ using a least-squares method to obtain $A$ and $C$. Information should therefore be encoded in the (previously marginalised) best-fit parameters as a function of $\Delta K$, and the suitability of both the model and the values of the global parameters can be tested by the fitting to $F_{H}$. Given the well-defined maximum of Figure 3(a), the model must be an excellent description of some, but not necessarily all, of the data. As mentioned previously, adsorbate interactions may be important on certain length scales, meaning that the least-squares fitting to the non-interacting analytic model will be less successful at the corresponding val- 

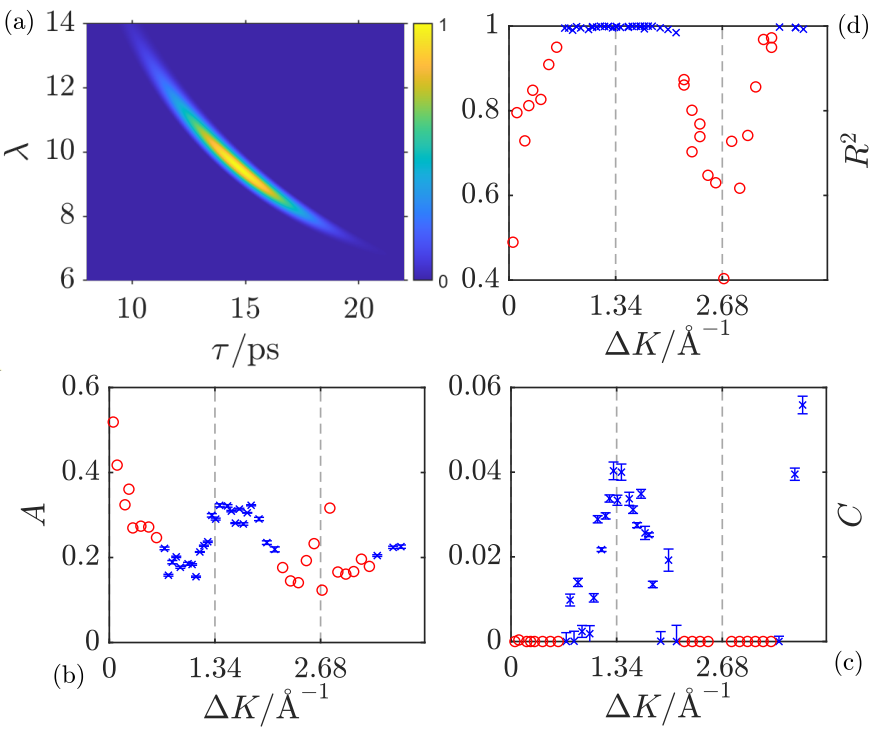

FIG. 3: (a) Relative probability generated by the marginalized Bayesian method, in $(\lambda, \tau)$ space, for the whole data set. The sharply peaked distribution indicates that the method is successful. (b) Fitted amplitude $A$, showing a clear peak. (c) Fitted constant $C$; the peak width is an indication of the size of the islands. (d) $R^{2}$ statistic from fitting $F_{H}$ to the data. $F_{H}$ and the global parameters provide a good fit to the experimental ISFs in the vicinity of the half-order position but are unsuitable elsewhere. All points associated with $R^{2}<0.98$ are plotted as red circles, with error bars omitted for clarity.

ues of $\Delta \mathbf{K}$. The values of $R^{2}$ therefore reveal the range of $\Delta \mathbf{K}$ values in which inter-adsorbate interactions are important. Plots of $A, C$, and the $R^{2}$ statistic as a function of $\Delta \mathbf{K}$ are presented in Figures $3(\mathrm{~b}),(\mathrm{c})$, and (d), respectively.

The background term $C$, which has units of polarization, is the residual elastic level of the ISF after the decay of inelastic and quasi-elastic processes. $C$ therefore includes contributions from parts of the system which are quasi-static relative to the correlation times probed. In particular, it is affected by slowly moving adsorbates and the periodic substrate which produces diffraction peaks in $C(\Delta \mathbf{K})$. A model can be applied to extrapolate the decay of an ISF in order to estimate $C$ for ISFs which are not fully decayed by the maximum accessible correlation time. In the present case, we have used the expression $F_{H}$.

A conventional diffraction experiment effectively integrates over all energies in the scattered beam and contains both elastic and inelastic components. However, as $C$ only contains a pseudo-elastic signal, it is effectively a time-resolved diffraction scan evaluated at a large correlation time 34. Such time-resolved scans have previously been obtained using time-of-flight helium atom scattering [35. With ${ }^{3} \mathrm{HeSE}$, thanks to the improved energy resolution, low energy inelastic contributions are filtered-out more efficiently, so the result more accurately reflects the quasi-static island structure. The position of the peak in $C(\Delta \mathbf{K})$ of Figure 3 (c) is consistent with clusters of $p(2 \times 2) \mathrm{O}$ observed on $\mathrm{Ru}(0001)$ at low temperatures and with the high-temperature diffraction scans we have measured. We therefore attribute the peak to scattering from quasi-static oxygen islands. Since background inelastic processes are absent from $C$, the mean cluster size can be estimated from the full-width halfmaximum (FWHM) of the peak [36, 37. We determine a value of $\approx 9 \AA$ (via $2 \pi / \delta \Delta K$, for FWHM $\delta \Delta K$ ). The finite size of and spread of energies in the beam also lead to broadening of the peak but are insignificant contributions here. We note that scaling $C$ to units of absolute intensity does not alter our conclusions or result in further insight. Overall, removing the quasielastic signal in order to obtain a pseudo-elastic diffraction scan, which is readily achievable using ${ }^{3} \mathrm{HeSE}$, has allowed us to study the in situ growth of nanoscopic islands in dynamic equilibrium.

$A$, the amplitude of the decay in polarization, can be considered independently and also contains valuable information regarding clustering. In general, $A$ relates to inelastic/quasielastic processes occurring over the measurement time. It is constant with $\Delta \mathbf{K}$ when isolated, non-interacting point particles are responsible for the diffusion signature. In contrast, truly immobile clusters should only produce an elastic signal which would appear as peaks in the constant $C(\Delta \mathbf{K})$. While we observe such a feature in $C$, we also resolve a peak in $A$ at roughly the half-order position, showing that the atomic diffusion and the evolution of the quasi-static clusters are strongly coupled at $T=850 \mathrm{~K}$. Detailed modelling of $A$, which is beyond the scope of this work, should shed light on the dynamical nature of oxygen islands. The fact that $A$ peaks at a value of $\Delta K$ slightly greater than $1.34 \AA^{-1}$ suggests that the particles prefer a separation less than $2 a$ and hence that the position of the minimum of the interadsorbate potential may also be reduced. The fcc site may be relevant in explaining the peak. We do not believe that $A$ is strongly influenced by the intensity scattering form factor, given that the form factor of isolated $\mathrm{O}$ atoms, which dominate the diffusion signature, is expected to decay monotonically [21, 38].

To test the hollow site model $F_{H}$ and the values of $\lambda=9.5 \pm 1$ and $\tau=14.3 \pm 2 \mathrm{ps}$ at different values of $\Delta \mathbf{K}$, we calculate the $R^{2}$ fitting statistic for each experimental ISF. The accuracy of the best-fit $A$ and $C$ can also be studied in this way. $R^{2}(\Delta \mathbf{K})$ is plotted in Figure $3(\mathrm{~d})$ and confirms the model explains the data very well near the half-order position, where the significant features in $A$ and $C$ are resolved (blue points). However, $R^{2}$ is low near $\Delta K=0$ and the $\mathrm{BZ}$ boundaries (red circles). In the SI, we illustrate that the experimental dephasing rate is greater than that predicted by theory in those regions. The model/global parameters therefore describe less well the ISFs corresponding to the red points. Multiple jumps 
in the system or inter-adsorbate interactions are most likely responsible for the deviation. Interpreting the data in terms of a multiple-jumps model similar to $f_{H}$ 39. results in unphysical values for the global parameters, as discussed in the SI. We therefore retain the single jump $f_{H}$ model here, stress that the model parameters describe the non-interacting part of the diffusion, and note that interactions are probably needed to fully explain certain parts of the data. Finally, excluding the ISFs with low $R^{2}$ values from the global analysis leads to negligible change in the global parameters and hence the features resolved in $A$ and $C$.

In summary, atomic diffusion in the low-coverage $\mathrm{O} / \mathrm{Ru}(0001)$ system has been measured at elevated temperatures using ${ }^{3} \mathrm{HeSE}$. Our results reveal the presence of oxygen clusters, in dynamic equilibrium with a gas of rapidly diffusing adatoms. We obtain a time-resolved diffraction scan by processing the dynamical data which allows us to estimate the mean size of the clusters. Analysis of the data within atomic jump diffusion models pro- vides detailed information on the energy landscape of the diffusing oxygen atoms. We have therefore demonstrated that ${ }^{3} \mathrm{HeSE}$ can be used to study islands at the onset of $2 \mathrm{D}$ surface growth, via the dynamic equilibrium that exists between clusters and diffusing precursors, at temperatures above the order-disorder phase transition.

* Supplementary Information contains references [22, 23, 29, 39, 45].

\section{ACKNOWLEDGMENTS}

The work was conducted at the Cambridge Atom Scattering Facility (CASF), with support from the Engineering and Physical Sciences Research Council (EPSRC) via grant $\mathrm{EP} / \mathrm{T} 00634 \mathrm{X} / 1$. Beam-time was funded by the Isaac Newton Trust, grant 17.37(j). The CASF management committee acknowledges both the EPSRC and the Herchel Smith Fund. J. K. gratefully acknowledges the EPSRC for a doctoral studentship.
[1] D. L. Duong, S. J. Yun, and Y. H. Lee, van der waals layered materials: opportunities and challenges, ACS nano 11, 11803 (2017).

[2] Z. Sun, A. Martinez, and F. Wang, Optical modulators with 2d layered materials, Nature Photonics 10, 227 (2016).

[3] S. M. Kim, A. Hsu, M. H. Park, S. H. Chae, S. J. Yun, J. S. Lee, D.-H. Cho, W. Fang, C. Lee, T. Palacios, et al., Synthesis of large-area multilayer hexagonal boron nitride for high material performance, Nature communications 6, 1 (2015).

[4] F. R. Eder, J. Kotakoski, U. Kaiser, and J. C. Meyer, A journey from order to disorder - atom by atom transformation from graphene to a $2 \mathrm{~d}$ carbon glass, Scientific reports 4, 4060 (2014).

[5] J. Lu, K. Zhang, X. F. Liu, H. Zhang, T. C. Sum, A. H. C. Neto, and K. P. Loh, Order-disorder transition in a twodimensional boron-carbon-nitride alloy, Nature communications 4, 1 (2013).

[6] L. Song, L. Ci, H. Lu, P. B. Sorokin, C. Jin, J. Ni, A. G. Kvashnin, D. G. Kvashnin, J. Lou, B. I. Yakobson, et al., Large scale growth and characterization of atomic hexagonal boron nitride layers, Nano letters 10, 3209 (2010).

[7] Z. Sun, Z. Yan, J. Yao, E. Beitler, Y. Zhu, and J. M. Tour, Growth of graphene from solid carbon sources, Nature 468, 549 (2010).

[8] F. M. Sapountzi, J. M. Gracia, C. K.-J. Weststrate, H. O. Fredriksson, and J. H. Niemantsverdriet, Electrocatalysts for the generation of hydrogen, oxygen and synthesis gas, Progress in Energy and Combustion Science 58, 1 (2017)

[9] E. Voloshina, N. Berdunov, and Y. Dedkov, Restoring a nearly free-standing character of graphene on $\mathrm{Ru}(0001)$ by oxygen intercalation, Scientific Reports 6 , 20285 (2016).

[10] T. E. Madey, H. A. Engelhardt], and D. Menzel, Adsorption of oxygen and oxidation of $\mathrm{CO}$ on the ruthenium
(001) surface, Surface Science 48, 304 (1975).

[11] J. Fuggle, T. Madey, M. Steinkilberg, and D. Menzel, Photoelectron spectroscopic studies of adsorption of CO and oxygen on $\mathrm{Ru}(001)$, Surface Science 52, 521 (1975).

[12] W. Kohn and K. H. Lau, Adatom dipole moments on metals and their interactions, Solid State Communications 18, 553 (1976)

[13] G. Alexandrowicz, A. P. Jardine, H. Hedgeland, W. Allison, and J. Ellis, Onset of 3D collective surface diffusion in the presence of lateral interactions: $\mathrm{Na} / \mathrm{Cu}(001)$, Phys. Rev. Lett. 97, 156103 (2006).

[14] A. Tamtögl, M. Sacchi, N. Avidor, I. Calvo-Almazán, P. S. M. Townsend, M. Bremholm, P. Hofmann, J. Ellis, and W. Allison, Nanoscopic diffusion of water on a topological insulator, Nature Communications 11 (2020).

[15] P. Piercy, K. De'Bell, and H. Pfnür, Phase diagram and critical behavior of the adsorption system $\mathrm{O} / \mathrm{Ru}(001)$ : Comparison with lattice-gas models, Phys. Rev. B 45, $1869(1992)$.

[16] C. Stampfl, S. Schwegmann, H. Over, M. Scheffler, and G. Ertl, Structure and stability of a high-coverage $(1 \times 1)$ oxygen phase on $\mathrm{Ru}(0001)$, Phys. Rev. Lett. 77, 3371 (1996)

[17] J. Wintterlin, J. Trost, S. Renisch, R. Schuster, T. Zambelli, and G. Ertl, Real-time stm observations of atomic equilibrium fluctuations in an adsorbate system: $\mathrm{O} / \mathrm{Ru}(0001))$, Surface Science 394, 159 (1997)

[18] S. Renisch, R. Schuster, J. Wintterlin, and G. Ertl, Dynamics of adatom motion under the influence of mutual interactions: $\mathrm{O} / \mathrm{Ru}(0001)$, Phys. Rev. Lett. 82, 3839 (1999)

[19] B. Holst, G. Alexandrowicz, N. Avidor, G. Benedek, G. Bracco, W. E. Ernst, D. Farías, A. P. Jardine, K. Lefmann, J. R. Manson, et al., Material properties particularly suited to be measured with helium scattering: Selected examples from $2 d$ materials, van der waals heterostructures, glassy materials, catalytic sub- 
strates, topological insulators and superconducting radio frequency materials, Physical Chemistry Chemical Physics (2021).

[20] P. Fouquet, A. Jardine, S. Dworski, G. Alexandrowicz, W. Allison, and J. Ellis, Thermal energy he 3 spinecho spectrometer for ultrahigh resolution surface dynamics measurements, Review of Scientific Instruments 76, 053109 (2005).

[21] A. P. Jardine, H. Hedgeland, G. Alexandrowicz, W. Allison, and J. Ellis, Helium-3 spin-echo: Principles and application to dynamics at surfaces, Progress in Surface Science 84, 323 (2009)

[22] S. Charkabortty and H. E. Grenga, Adsorption of carbon monoxide on ruthenium, Journal of Applied Physics 44, 500 (1973), https://doi.org/10.1063/1.1661917.

[23] G. Lauth, E. Schwarz, and K. Christmann, The adsorption of hydrogen on a ruthenium (1010) surface, The Journal of Chemical Physics 91, 3729 (1989), https://doi.org/10.1063/1.456856

[24] C. T. Chudley and R. J. Elliott, Neutron scattering from a liquid on a jump diffusion model, Proceedings of the Physical Society 77, 353 (1961)

[25] F. E. Tuddenham, H. Hedgeland, A. P. Jardine, B. A. Lechner, B. J. Hinch, and W. Allison, Lineshapes in quasi-elastic scattering from species hopping between non-equivalent surface sites, Surface Science 604, 1459 (2010)

[26] P. G. de Gennes, Liquid dynamics and inelastic scattering of neutrons, Physica 25, 825 (1959)

[27] S. P. Rittmeyer, D. J. Ward, P. Gütlein, J. Ellis, W. Allison, and K. Reuter, Energy dissipation during diffusion at metal surfaces: Disentangling the role of phonons versus electron-hole pairs, Phys. Rev. Lett. 117, 196001 (2016)

[28] G. Alexandrowicz, P. R. Kole, E. Y. M. Lee, H. Hedgeland, R. Ferrando, A. P. Jardine, W. Allison, and J. Ellis, Observation of uncorrelated microscopic motion in a strongly interacting adsorbate system, Journal of the American Chemical Society 130, 6789 (2008), pMID: 18457388, https://doi.org/10.1021/ja800118x

[29] P. S. M. Townsend, Diffusion of Light Adsorbates on Transition Metal Surfaces, Ph.D. thesis, University of Cambridge (2018).

[30] B. A. J. Lechner, P. R. Kole, H. Hedgeland, A. P. Jardine, W. Allison, B. J. Hinch, and J. Ellis, Ultra-high precision determination of site energy differences using a bayesian method, Phys. Rev. B 89, 121405 (2014)

[31] E. M. McIntosh, K. T. Wikfeldt, J. Ellis, A. Michaelides, and W. Allison, Quantum effects in the diffusion of hydrogen on $\mathrm{Ru}(0001)$, The Journal of Physical Chemistry Letters 4, 1565 (2013), pMID: 24920996, https://doi.org/10.1021/jz400622v

[32] J.-Q. Cai, H.-J. Luo, X.-M. Tao, and M.-Q. Tan, Initial subsurface incorporation of oxygen into
$\mathrm{Ru}(0001)$ : A density functional theory study, ChemPhysChem 16, 3937 (2015), https://chemistryeurope.onlinelibrary.wiley.com/doi/pdf/10.1002/cphc.201500681

[33] C. Stampfl and M. Scheffler, Theoretical study of O adlayers on Ru(0001), Phys. Rev. B 54, 2868 (1996).

[34] K. A. Stoerzinger, Nanoscale Dynamics of Benzene and Naphthalene on Graphene/Ru(0001), Master's thesis, University of Cambridge (2011).

[35] C. Meli, E. Greene, G. Lange, and J. Toennies, Evidence for an order-order transition on the ge (111) surface near $1050 \mathrm{k}$ from high-resolution helium atom scattering experiments, Physical review letters 74, 2054 (1995).

[36] N. Camillone, C. E. Chidsey, G.-y. Liu, T. Putvinski, and G. Scoles, Surface structure and thermal motion of n-alkane thiols self-assembled on au (111) studied by low energy helium diffraction, The Journal of chemical physics 94, 8493 (1991).

[37] G. Comsa, Coherence length and/or transfer width?, Surface Science 81, 57 (1979).

[38] D. J. Ward, A. Raghavan, A. Tamtögl, A. P. Jardine, E. Bahn, J. Ellis, S. Miret-Artès, and W. Allison, Interadsorbate forces and coherent scattering in helium spinecho experiments, Physical Chemistry Chemical Physics (2021).

[39] P. S. M. Townsend and N. Avidor, Signatures of multiple jumps in surface diffusion on honeycomb surfaces, Phys. Rev. B 99, 115419 (2019).

[40] A. Raghavan, L. Slocombe, A. Spreinat, D. J. Ward, W. Allison, J. Ellis, A. P. Jardine, M. Sacchi, and N. Avidor, Alkali metal adsorption on metal surfaces: new insights from new tools, Physical Chemistry Chemical Physics (2021).

[41] B. Poelsema and G. Comsa, Scattering of thermal energy atoms: from disordered surfaces, Vol. 115 (Springer, 2006).

[42] E. McIntosh, P. Kole, M. El-Batanouny, D. Chisnall, J. Ellis, and W. Allison, Measurement of the phason dispersion of misfit dislocations on the au (111) surface, Physical review letters 110, 086103 (2013).

[43] M. Gladys, A. El-Zein, A. Mikkelsen, J. Anderson, and G. Held, Modifying the adsorption characteristics of water on ru $\{0001\}$ with preadsorbed oxygen, Physical Review B 78, 035409 (2008).

[44] G. Praline, B. Koel, H.-I. Lee, and J. White, Incorporation of oxygen chemisorbed on $\mathrm{Ru}(001)$, Applications of Surface Science 5, 296 (1980).

[45] N. Avidor, H. Hedgeland, G. Held, A. P. Jardine, W. Allison, J. Ellis, T. Kravchuk, and G. Alexandrowicz, Highly proton-ordered water structures on oxygen precovered $\mathrm{Ru}(0001)$, The Journal of Physical Chemistry A 115, 7205 (2011) pMID: 21504173, https://doi.org/10.1021/jp200221b. 